\title{
Metrics and Analyses in The Test Phase of Large-Scale Software
}

\author{
E. Obara, T. Kawasaki, Y. Ookawa, and N. Maeda \\ NTT Telecommunications Software Headquarters
}

\author{
2-1-1 Nishi-Gotanda, Shinagawa-Ku, Tokyo 141, Japan \\ Tel. $+81-3-5487-9143 \quad$ Fax. $+81-3-5487-9790$ \\ E-mail: obara.eiji@d70.tsh.cae.ntt.jp
}

\begin{abstract}
To predict the time needed for a test case, four factors that characterize test cases were extracted and the time needed for actual test cases was measured. This paper describes (1) the formula for predicting the number of test MM (Man-Months) from these factors, (2) the development of the skill curve in the test phase, and (3) the modeling of the evolution process for groups of software engineers. These results can be used to predict and improve test productivity. This paper also reports how to extract factors effective in characterizing the testing processes and measuring the MM of test cases. It then discusses a prediction formula, efficient curricula derived from the skill curve in the test phase, and a model of the evolution of software development factories.
\end{abstract}

keywords
test phase, metrics, measurement, skill curve, model, estimate, curriculum

\section{Introduction}

Large-scale switching software must have a failure rate so low that the software is out of service less than one hour in 20 years (Ishii, 1991.) The quality of the software also needs to be improved in order to maintain such a low failure rate and to enable $200 \mathrm{~K}$ lines for new services to be accommodated annually throughout the software lifetime. Because the number of these additional services is huge, they will eventually amount to $15 \%$ of the entire system. It is therefore especially important to increase the productivity of the test phase.

Few studies have focused on the productivity of the test phase, possibly because the skills in testing factories are extremely diversified and the application of test-equipment is very complex. Very little has been done to identify the relationship between the test productivity and the time required to develop the technical skills of testing engineers.

Several metric methods have been used to measure the productivity and complexity of software testing, but according to Beizer (1990) none have been successful. Many methods have been suggested to measure the entire life-cycle of the software development, but none have been found to be particularly effective for a specific phase, such as the test phase. 
McCabe (1976) studied the measurement of program complexity. The complexity measured in his method is related to the productivity of the design and implementation phases but is not directly related to the productivity in the test phase. Though the productivity in the test phase depends on whether or not the test case needs terminal simulators, McCabe dealt with the complexity of the program by examining the program structure.

Walston and Felix (1977) measured 29 factors related to the complexity of the user interface between the software system and the user, and they discovered the relationship between these factors and software productivity. DeMarco $(1982$, ) however, pointed out the possible influence of measured engineers upon the measurement results. Measured engineers tend to think that their poor productivity is due to the complexity of the user interface and consider this complexity to be greater than it actually is. This is a psychological aspect of the measurement of complicated phenomena, and the metrics factors must therefore be selected independently of the desires of the testing engineers.

The present study is therefore intended to shed light on this complex issue, with a view to improving the productivity of the software in the test phase. To identify the issues and problems in test productivity, the authors applied the quantification 1 analysis of test productivity. Since software development is undertaken in the ten NTT factories scattered all over the country, the complexity of testing the new services to be conducted in the test phase was addressed first. Then the ten factories were analyzed so that they could be categorized with regard to factors most likely to affect test productivity. Each of the factories was then categorized into a model and then curricula for educating unskilled engineers were developed.

To further improve the data base, care was taken to avoid the influence of measurement engineers when extracting factors in the test phase.

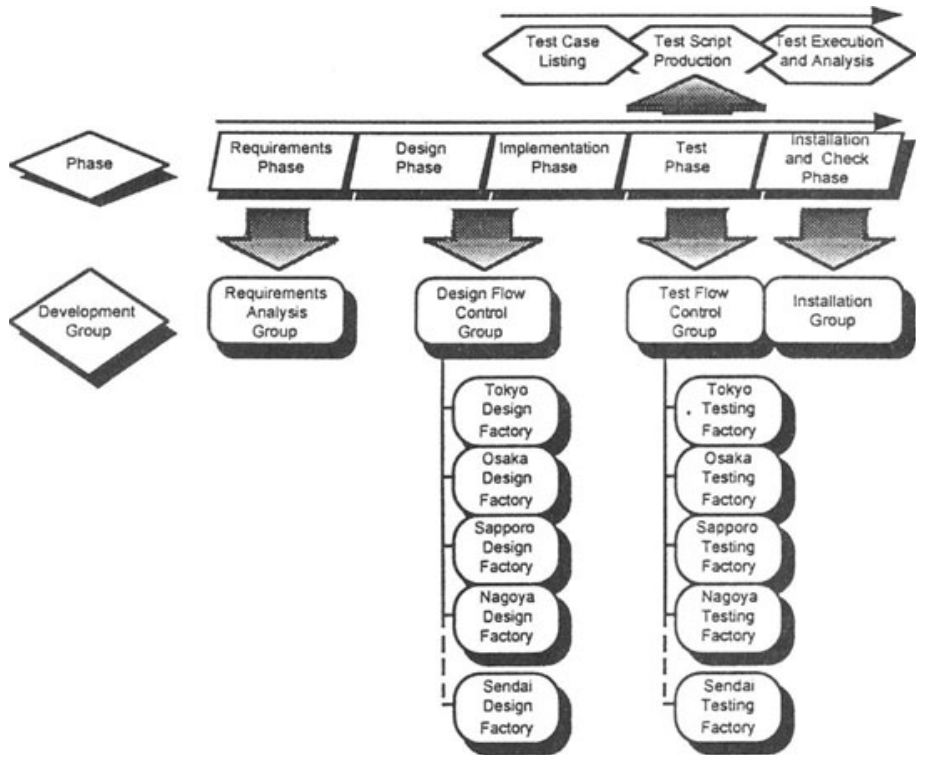

Figure 1 Flow and organization of D70 switching software development. 


\section{Environment and Classification Method of Test Cases}

\subsection{Environment of Switching Software Development}

In the case of the D70, one of NTT's major switching machines, 60-80 new services that are equivalent to about $200 \mathrm{~K}$-lines are added annually. The total volume of new services amounts to about $15 \%$ of the entire D70 system, and about 700 software engineers are engaged in developing these services.

The D70 development system is divided into several phases. In the test phase, about 300 testing engineers are charged with work ranging from Unit Testing to Installation Checking. The design factories and the testing factories are located in ten major cities in Japan. This nationwide development system enables NTT to supply all factories with many well-selected engineers.

New service features to be added are thoroughly studied and defined by the requirement analysis group and are then, for detailed design, sent to any of the design factories. The design groups are charged with all the tasks from basic design to compilation and linkage. The subsequent test phase may be performed at any testing factory.

Table 1 Factors and their values

\begin{tabular}{|c|c|c|c|c|c|}
\hline $\begin{array}{c}\text { Factors Identifying } \\
\text { the Services }\end{array}$ & \multicolumn{5}{|c|}{ Factor Values \& Items } \\
\hline Service IDs & \multicolumn{5}{|c|}{$\begin{array}{l}\text { 3ba01, 3ba02, 3ba03, 3ba04, 3ba05, 3ba06, 3ba07, 3ba09, 3ba11, } \\
\text { 3ba12, 3ba13, 3ba14, 3ba16, 3ba17, 3ba18, 3ba19, 3ba21, 3ba22, } \\
\text { 3ba23, 3ba25, 3ba27, 3ba50, 3ba51, 3ba60, 3ba61, 3ba62, 3ba63, } \\
\text { 3ba64, 3bc01, 3bc02, 3bc50, 3bd05, 3bd30, 3bd31, 3bk01, 3bl01, } \\
\text { 3bp01, 3bz50 }\end{array}$} \\
\hline Classes of Services & \multicolumn{3}{|c|}{$\begin{array}{l}\text { OriginatingCall Service } \\
\text { Restart Feature } \\
\text { Command service } \\
\text { Common Channel SignalFeature } \\
\text { Diagnosis Feature } \\
\end{array}$} & \multicolumn{2}{|c|}{$\begin{array}{l}\text { Terminating Call Service } \\
\text { Construction Feature } \\
\text { Fault Recognition Feature } \\
\text { System Configuration Feature }\end{array}$} \\
\hline Other Node Simulator & \multicolumn{3}{|l|}{ Needed } & \multicolumn{2}{|c|}{ Not Needed } \\
\hline Program Route & \multicolumn{3}{|c|}{ Normal Route } & \multicolumn{2}{|c|}{ Abnormal Route } \\
\hline Office Data Change & \multicolumn{3}{|c|}{ Change } & \multicolumn{2}{|c|}{ No Change } \\
\hline New Equipment & \multicolumn{3}{|l|}{ New } & \multicolumn{2}{|l|}{ Old } \\
\hline Software Reusability & \multicolumn{3}{|l|}{ Reusable } & \multicolumn{2}{|c|}{ New Software } \\
\hline Method of Testing & \multicolumn{3}{|l|}{ Automation } & \multicolumn{2}{|l|}{ Manual } \\
\hline Terminal Simulator & \multicolumn{3}{|l|}{ Needed } & \multicolumn{2}{|c|}{ Not Needed } \\
\hline Factories & $\begin{array}{l}\text { Hiroshima } \\
\text { Nagano } \\
\text { Sendai }\end{array}$ & $\begin{array}{l}\text { Kanazawa } \\
\text { Nagoya } \\
\text { Tokyo }\end{array}$ & \multicolumn{2}{|c|}{$\begin{array}{l}\text { Kumamoto } \\
\text { Osaka }\end{array}$} & $\begin{array}{l}\text { Matsuyama } \\
\text { Sapporo }\end{array}$ \\
\hline Skill of Test Script & $<1$ & $1-2$ & \multicolumn{2}{|c|}{$3-4$} & $>5$ \\
\hline Skill of Executing Test & $\begin{array}{l}<1 \\
<1 \& 3-4 \\
<1 \&>5\end{array}$ & $\begin{array}{l}1-2 \\
1-2 \& 3-4 \\
1-2 \&>5\end{array}$ & \multicolumn{2}{|c|}{$\begin{array}{l}<1 \& 1-2 \\
<1 \text { \& } 1-2 \text { \& } 3-4\end{array}$} & $\begin{array}{l}3-4 \\
>5\end{array}$ \\
\hline
\end{tabular}

The flow of the test phase consists of (1) the extraction of the test cases from the 
specification of the new services to be added and their codes, (2) the production of test scripts for each test case, (3) the execution of test cases, and (4) the analysis of the results of the executed test cases. The production of the test scripts and the execution of the test cases make up approximately $60 \%$ of the tasks of the entire test phase. Accordingly, this paper concerns mainly these two tasks (Figure 1.)

\subsection{Classification Method of Test Cases}

The complexity in the test phase depends not on the structure of the software program but on a complicated test system consisting of numerous and diversified test elements with different levels. To work out a new rational test system, we initiated the study by sequentially raising the following basic questions:

- Does a test case need new equipment?

- Does the protocol with other nodes need any changes?

- Does a test case contain features of originating or terminating call service?

Considering past experience in software development, we chose the 12 factors listed in Table 1 to characterize the structure of the test cases.

Table 2 Example of Test Case IDs vs. factors

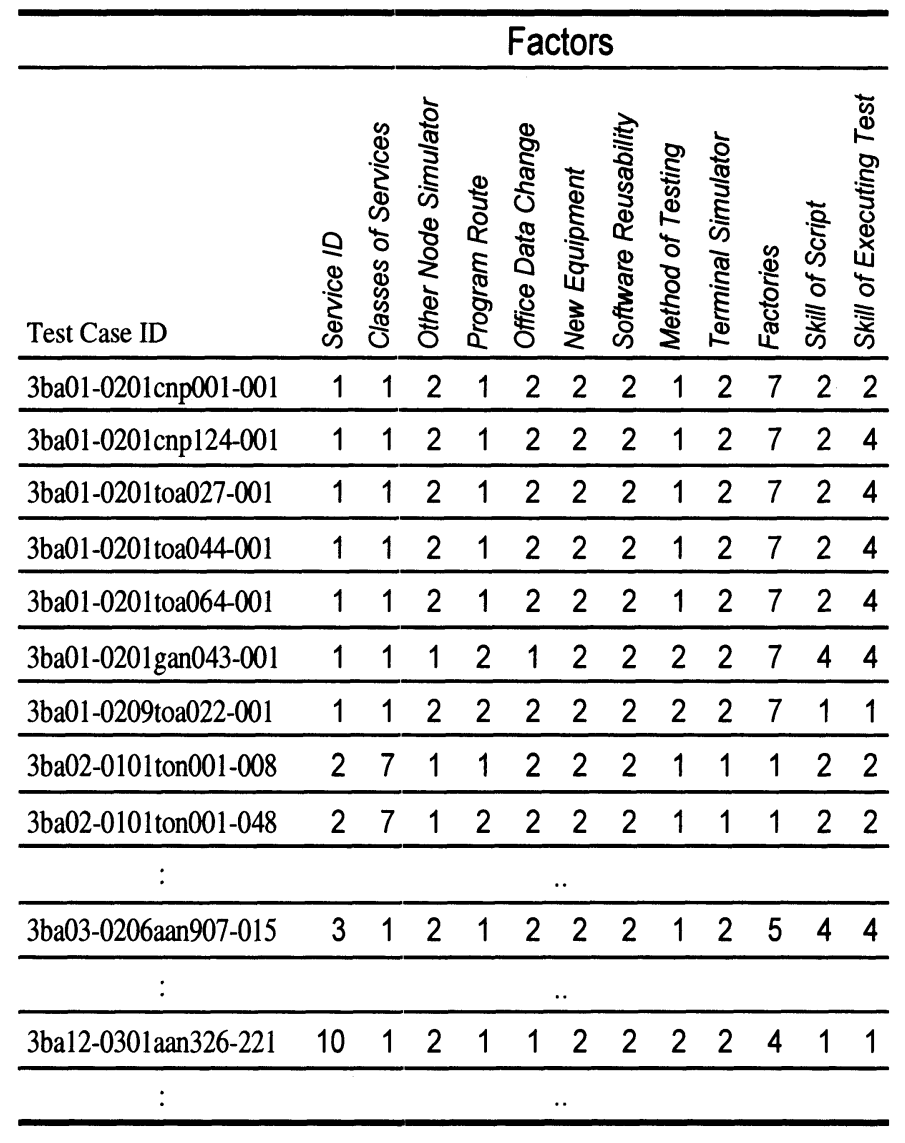


These items are numbered in accordance with quantification 1 analysis. As can be seen in Table 2, for test case ID 3ba01-0201 cnp001-001, the entry in the column for the Classes of Services factor is 1 and so this test case has the feature of Originating Call Services.

Table 3 Example of GTC data

\begin{tabular}{|c|c|c|c|c|c|c|c|c|c|c|c|c|c|c|c|}
\hline \multirow[b]{2}{*}{ 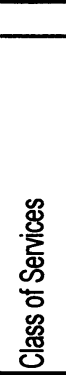 } & \multirow[b]{2}{*}{ 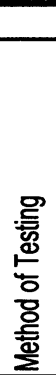 } & \multicolumn{4}{|c|}{ GTC ID } & \multicolumn{5}{|c|}{ Test Script (min.) } & \multicolumn{5}{|c|}{ Test Execution (min.) } \\
\hline & & $\begin{array}{l}\text { 흥 } \\
\text { क् } \\
\text { 흫 }\end{array}$ & 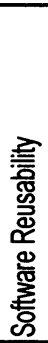 & 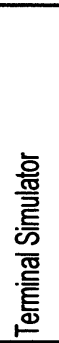 & 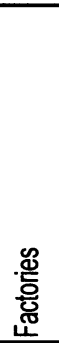 & 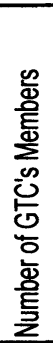 & 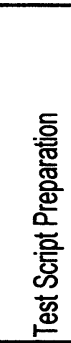 & 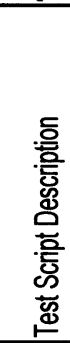 & $\begin{array}{l}\stackrel{\infty}{\Phi} \\
\text { D }\end{array}$ & 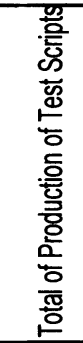 & 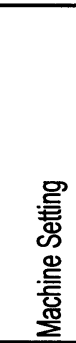 & 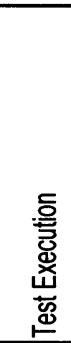 & 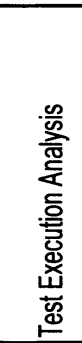 & 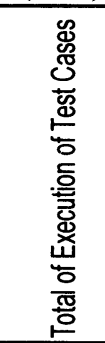 & 㞼 \\
\hline 1 & 1 & 2 & 2 & 2 & 7 & 11 & 17.0 & 36.6 & 1.3 & 54.9 & 30.0 & 69.3 & 12.6 & 111.9 & 166.8 \\
\hline 1 & 2 & 2 & 2 & 2 & 7 & 6 & 36.7 & 34.2 & 6.7 & 77.6 & 15.2 & 30.0 & 10.8 & 56.0 & 133.6 \\
\hline \multirow[t]{2}{*}{7} & $\overline{1}$ & 2 & 2 & 1 & 1 & 9 & 23.5 & 21.4 & 1.3 & 46.2 & 15.2 & 26.4 & 13.7 & 55.3 & 101.5 \\
\hline & \multicolumn{3}{|c|}{$:$} & & & & & \\
\hline \multirow[t]{2}{*}{7} & 1 & 2 & 2 & 1 & 2 & 5 & 5.4 & 18.1 & 0.0 & 23.5 & 20.4 & 9.0 & 7.5 & 36.9 & 60.4 \\
\hline & \multicolumn{3}{|c|}{$:$} & & & & \multicolumn{4}{|c|}{ : } & \\
\hline \multirow[t]{2}{*}{7} & 2 & 2 & 1 & 1 & 6 & 6 & 26.5 & 39.2 & 0.0 & 65.7 & 15.2 & 30.5 & 0.0 & 45.7 & 111.4 \\
\hline & & : & & & & & & : & & & & & & & \\
\hline
\end{tabular}

Each factor consists of several items, and one item characterizes a test case. For example, for New Equipment there are two items: New and Old. A group of test cases that have common items is called a GTC (Group of Test Cases). For example, a GTC may consist of 11 test cases with common items. They are Originating Call Services for the factor Classes of Services, Automation for Method of Testing, 1-2 (Year) for Skill of Test Script, New Software for Software Reusability, and so on. These test cases belong to the same GTC because they have items common to each of the factors. A test case is classified into a GTC for a set of items. If the MM (Man-Months) needed for a test case is measured, then the test productivity of the GTC may be estimated. In applying the GTC method, the productivity of a test may be predicted. This approach is called the GTC method.

Table 3 is an example of the data actually measured. According to this table, the GTC is identified by six factors: Classes of Services, Method of Testing, Skill of Script, Software Reusability, Terminal Simulator, and Factories. Each row of Table 3 indicates a GTC and the values of its productivity, which are the averages of test cases that belong to the GTC. The GTC-ID of the first row in Table 3 is 1-1-2-2-2-7. Each number represents items for each of the six factors.

In Table 3, six factors and their items provide the scheme of the GTCs. Accordingly, it will become possible to predict how many factors and their items will provide an accurate scheme of the GTCs. For example, if the 12 extracted factors are reduced to ten factors, the scheme of the GTCs will change. And the scheme of the GTCs will also change if the nine items of Classes of Services increase to 11.

The scheme must be adjusted to obtain the best prediction.

Test Case/MM (Man-Months), on how many test-cases a testing engineer can handle in one month, is used as a unit of productivity in the test phase. We chose Test Case/MM as an index of productivity in the test phase for two reasons: One is that the performance time of test cases is easy to measure, and the other is that Test Case/MM is 
nearly equivalent to Function Point/MM (since one test case checks about one function). In other words, the number of test cases is closely related to the number of function points.

Figure 2 Example of worksheet.

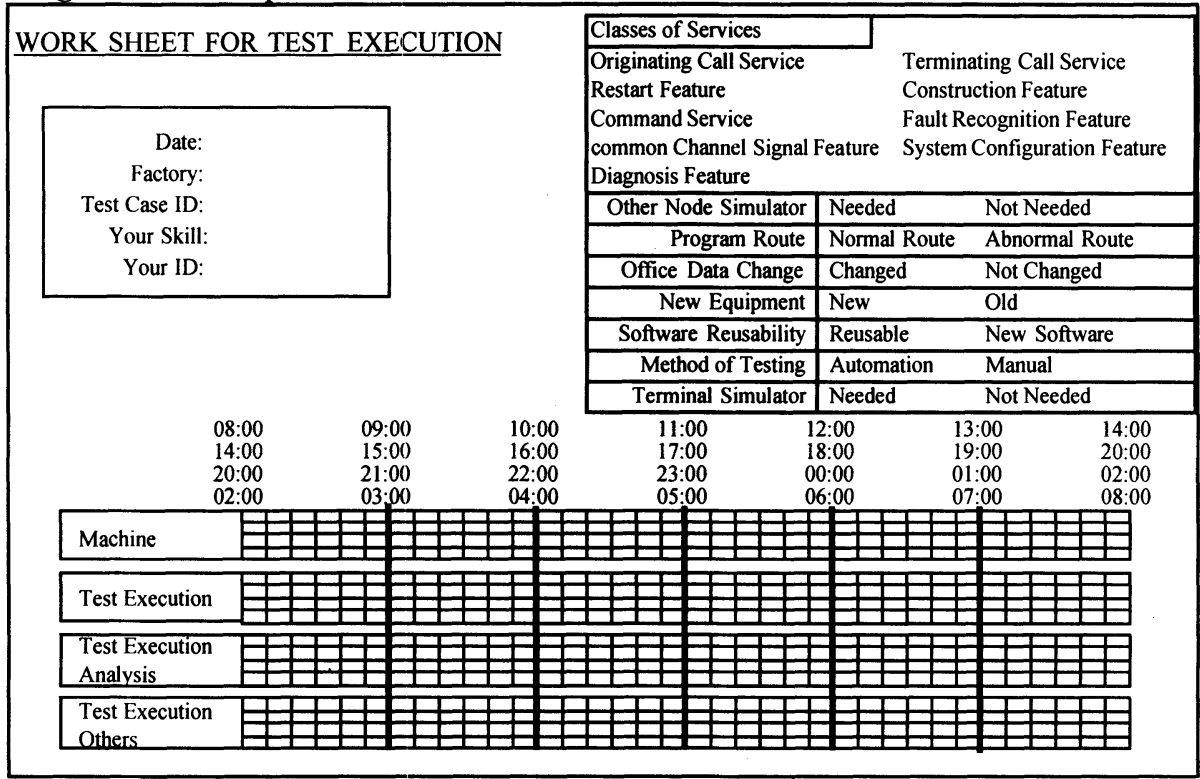

\section{Measurement in Factories}

The measurement process has four steps: (1) the selection of the test cases to be measured, (2) the examination of the GTCs, (3) measurement of MM for each test case, and (4) the gathering and analysis of the MM data.

The details of these steps are as follows: (1) In selecting test cases to be measured, 1200 of the entire 29000 cases were selected. The 29000 test cases were numbered, and then 1 was selected from every 24 . (2) The 1200 selected were examined to determine the GTC of each. Testing engineers to whom selected test cases were to be assigned were asked to fill out a worksheet (Figure 2) every day during the test period. The worksheet contained columns for the MM of the test case, the items of the test case, and information about the testing engineer (e.g., engineer's skill and the factory he belongs to). (3) Testing engineers measured the MM of their test cases during the period from October 1993 to April 1994. (4) The worksheets were collected at the end of every month and thoroughly checked to ensure their reliability.

\section{Analysis}

The measurements described in sections 2 and 3 made it possible to evaluate the measurements in the test phase in quantifiable terms and also enabled the following three analyses:

1. The formula of the MM as a function of the items of each test case;

2. Determining what kind of curriculum is effective for improving test skills; and 
3. The development of a model for the evolution of factories.

\subsection{MM as a Function of Test Case Items}

The formula for MM as a function of the items of each test case was derived by applying quantification 1 analysis (Honda, 1993.) 1,200 test cases were classified into 160 GTCs provided with 12 factors. Logically speaking, 12 factors can make more than 160 GTCs, but many of them have no test cases. The MM of GTCs that are vacant, however, can be estimated by applying quantification 1 analysis.

Quantification 1 analysis is not strictly accurate since there are more factors than are necessary. The number of factors and their items must be properly selected for accurate prediction. In this study, 12 factors were selected, which resulted in the coefficient of determination relating Total Test Time (Total time of the time of the production of a test script and the execution of a test case) to the 12 factors being about $12 \%$. The coefficient of determination to predict Total Test Time from the 12 factors and 85 items and samples of 1,200 test cases is not sufficient for accurate prediction because the itemization of the individual factors was too detailed. According to the quantification 1 analysis, ineffective factors can be eliminated and items that have few members can be combined into one item for accurate prediction. The 12 factors were therefore reduced to the following seven factors:

1. Classes of Services,

2. Method of Testing,

3. Software Reusability,

4. Terminal Simulator,

5. Factories,

6. Skill of Test Script, and

7. Ratio of Test Script Production to Execution (RPE.)

Ratio of Test Script Production to Execution (RPE), indicating the ratio of the time spent producing the test script to the time spent executing the case, gives us Test Style. Both producing a test script and executing the case are closely related to the complexity of the case. For example, a test case that is assigned to a person who is precise in running tests tends to have a large RPE value. And when a testing engineer is not well prepared for the test, the RPE of his test cases may be smaller than that of others.

Although RPE was not included in the 12 factors selected in the study, it was treated as an example of the factors that indicate the test style. Of the 12 factors and their items selected at the onset of the study, some were found not to contribute adequately to the test productivity. This led to the reexamination of the number of factors and their items to be further studied, and a reduction of the 12 factors to 7 by combining some and discarding others. There were some GTCs that have few test cases, and some test cases that showed abnormal results were ignored. Some similar items, such as some factories that bear resemblance in their environments and testing engineers, are combined to increase their contribution to the prediction. These were cases that;

1. had less than five Test Cases in a GTC, and

2. had Total Test Time (for production of the test script and execution of the test case) of more than 300 minutes.

3. Furthermore, three factories (Kumamoto, Matsuyama, and Sapporo) were integrated into one, and

4. two factories (Nagoya and Tokyo) were integrated into one.

As a result of this reexamination process, 55 GTCs were left being classified by seven factors and integrated items. By calculating the mean value of $\mathrm{MM}$ for test cases in each GTC and analyzing them by applying quantification 1 analysis and focusing on the four 
factors as listed below, a formula for accurate prediction could be derived.

1. Method of Testing

2. Software Reusability

3. Factories

4. Ratio of Time of the Production of the Test Script $\left(\mathrm{T}_{\mathrm{ps}}\right)$ to Time of the Execution of the Test Cases $\left(\mathrm{T}_{\mathrm{ec}}\right)$.

The coefficient of determination for the four factors is $58.4 \%$. The test productivity was then obtained using the following formula:

$\mathrm{T}=40.1 \times \mathrm{RPE}+\left[\begin{array}{llllll}0 & 114.0 & 41.9 & 82.6 & 48.6 & 57.9\end{array}\right]\left[\begin{array}{l}\mathrm{F}_{1} \\ \mathrm{~F}_{2} \\ \mathrm{~F}_{3} \\ \vdots \\ \mathrm{F}_{\mathrm{n}} \\ \vdots\end{array}\right]$

$+\left[\begin{array}{ll}0 & 23.8\end{array}\right]\left[\begin{array}{l}\mathrm{S}_{1} \\ \mathrm{~S}_{2}\end{array}\right]+\left[\begin{array}{ll}0 & -37.6\end{array}\right]\left[\begin{array}{l}\mathrm{M}_{1} \\ \mathrm{M}_{2}\end{array}\right]$,

where $T$ is Total Test Time $\left(T_{p s}+T_{e c}\right), R P E$ is the Ratio of $T_{p s}$ to $T_{e c}, F$ is the vector that represents Factory (e.g., $\mathbf{F}=\left[\begin{array}{c}1 \\ 0 \\ : \\ 0\end{array}\right]$ means Hiroshima, $\mathbf{F}=\left[\begin{array}{c}0 \\ 1 \\ : \\ 0\end{array}\right]$ means Kanazawa), $\mathbf{S}$ shows whether software is reusable or not (e.g., $\mathbf{S}=\left[\begin{array}{l}1 \\ 0\end{array}\right]$ means reusable and $\mathbf{S}=\left[\begin{array}{l}0 \\ 1\end{array}\right]$ means new software), and $\mathbf{M}$ shows the method of the test (e.g., $\mathbf{M}=\left[\begin{array}{l}1 \\ 0\end{array}\right]$ means automation test and $\mathbf{M}=\left[\begin{array}{l}0 \\ 1\end{array}\right]$ means manual test ) .

For instance, if a test case uses an automation method and its software is not reusable and if it is executed at Hiroshima factory and its RPE is equal to 1.2, the MM of the test case can be predicted by

$$
\mathrm{T}=71.9 \pm 11.3 \text { (min.) }
$$

Thus, the formula and the model for the prediction of the productivity in the test phase were obtained. In this model, four factors characterize the test phase and the necessary man-power can be estimated. According to estimated the work load, each of the factories and testing engineers will be re-assigned with the proper amount of tasks. 


\subsection{Effective Curriculum for Improving Test Skills}

Factor Skill of Production of Test Script and Skill of Execution of Test Case indicate the skill. Figures 3 and 4 show the relationship between the skill and the test productivity.

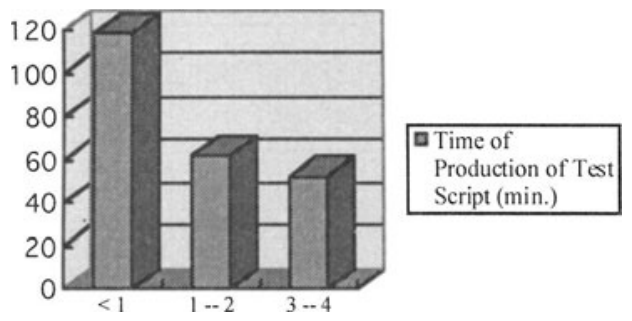

Figure 3 Script skill vs. time of production of test script.

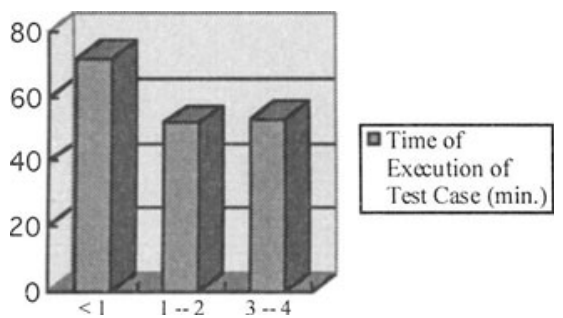

Figure 4 Execute skill vs. time of execution of test case.

These figures show that after one year of training, untrained engineers have acquired skills equivalent to those of engineers with four years of experience. In this connection, it should be noted that one year corresponds to the period of two life-cycles of new services.

Inspecting Figures 3 and 4, we should note that (1) the efficiency and effectiveness of on-the-job-training could be best achieved by selecting the engineers with less than one year of experience as the target group, and (2) to improve the testing skills of engineers with more than one year of experience, we need to develop different curricula.

\subsection{Model of Evolution of Factories}

This section addresses the issues related to the model for the evolution of factories, analyzing qualitatively the relationship between factors and the time required for Production of Test Script and for Execution of Test Case.

The two factors that have the largest F-values with regard to the Time of Production of Test Script $\left(\mathrm{T}_{\mathrm{ps}}\right)$ and the Time of Execution of a test case $\left(\mathrm{T}_{\mathrm{ec}}\right)$ were selected. According to the data listed in Tables 4 and 5, these factors are Skill of Test Script and Classes of Services. 
Table 4 Influence of factors on $T_{p s}$.

\begin{tabular}{lr}
\hline Factors & F-Value \\
\hline Skill of Test Script & 25.6 \\
\hline Terminal Simulator & 13.7 \\
\hline Software Reusability & 13.6 \\
\hline Method of Testing & 9.7 \\
\hline Classes of Services & 5.9 \\
\hline
\end{tabular}

Table 5 Influence of factors on $\mathrm{T}_{\text {ec }}$.

\begin{tabular}{lr}
\hline Factors & F-Value \\
\hline Classes of Services & 8.2 \\
\hline New Equipment & 6.5 \\
\hline Skill of Executing Test & 6.3 \\
\hline
\end{tabular}

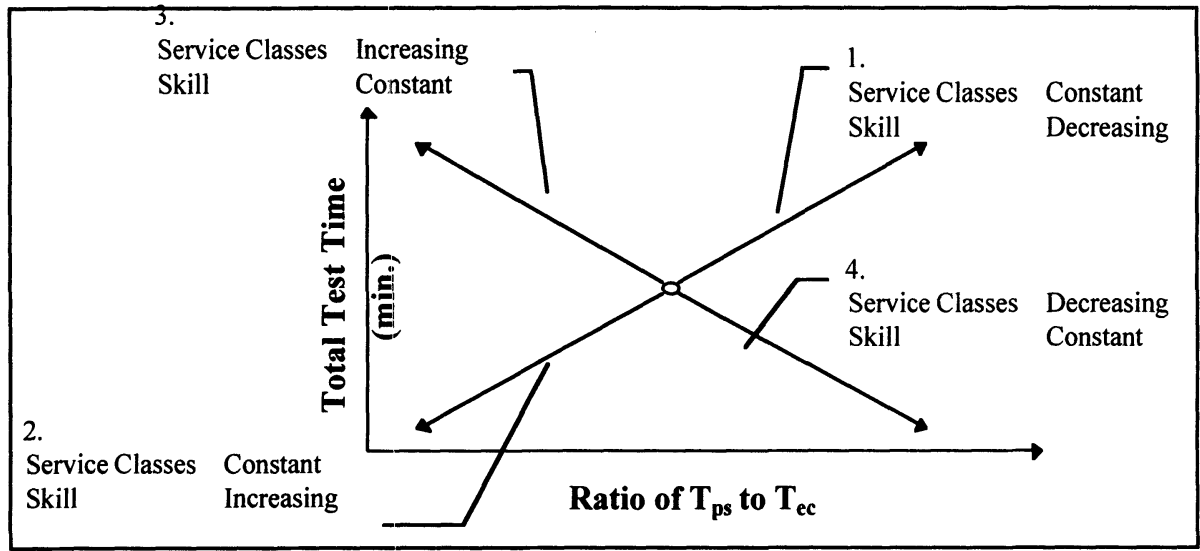

Figure 5 Ratio of $\mathrm{T}_{\mathrm{ps}}$ to $\mathrm{T}_{\mathrm{ec}}$ vs. Total Test Time.

Figure 5 illustrates the relationship between Total Test Time and RPE. Here $R P E=\frac{T_{p c}}{T_{e c}}$, where $T_{p s}$ means Time of Production of Test Scripts and depends mostly on Skill of Production of Test Script and $\mathrm{T}_{\mathrm{ec}}$ means Time of Execution of Test Cases and depends mostly on Classes of Services.

The hypothesis, that in order to examine how the point of a test case moves as the items of a test case change under the following rules, is considered.

1. Classes of Services constant, Skill of Production of Test Script decreasing. RPE increases because $T_{p s}$ (the numerator of RPE) is more sensitive than $\mathrm{T}_{\text {ec }}$ (the denominator of RPE). Total Test Time also increases. Therefore, the point moves toward the upper right.

2. Classes of Services constant, Skill of Production of Test Script increasing. Both RPE and Total Test Time decrease. Therefore, the point moves toward the lower left.

3. Classes of Services increasing, Skill of Production of Test Script constant. Because $T_{e c}$ (the denominator of RPE) is more sensitive than $T_{p s}$ (the numerator of RPE) RPE decreases, and Total Test Time increases. Therefore, the point moves toward the upper left.

4. Classes of Services decreasing, Skill of Production of Test Script constant. RPE increases and Total Test Time decreases. Therefore, the point moves toward the lower right.

In Figure 6, the values of $T_{p s}$ and $T_{e c}$ obtained for each of the factories are plotted against the scale of RPE vs. Total Test Time. 


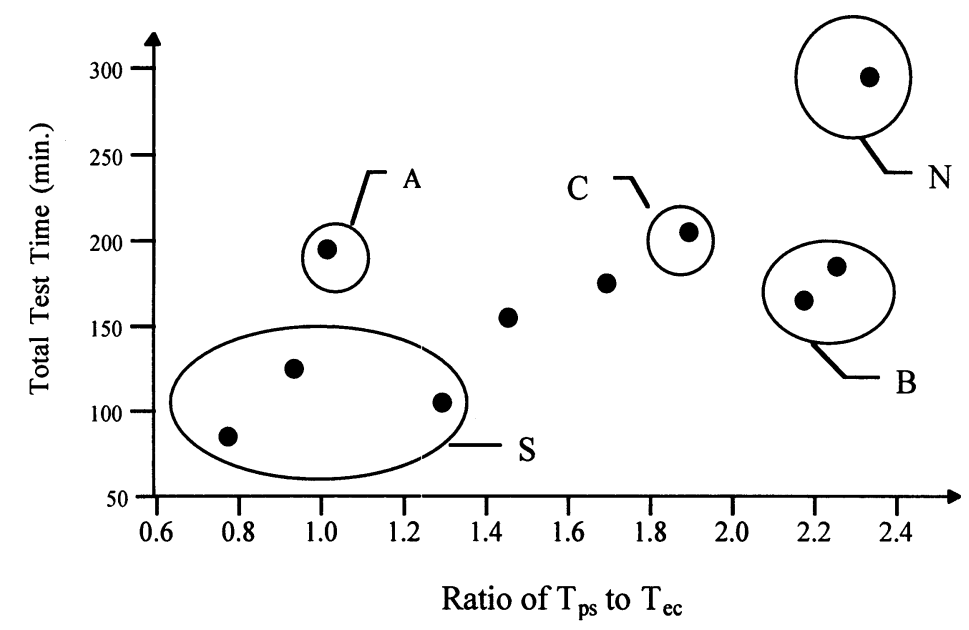

Figure 6 Ratio of $T_{p s}$ to $T_{e c} v s$. Total Test Time in factories.

While some factories are newly built and the overall skills there are yet to be improved, others factories are old and skills there are well established. In this paper, these two groups of factories are called the NFG (New Factories Group) and the SFG (Skilled Factories Group). Many engineers in the NFG are newcomers and unskilled whereas many engineers in the SFG are experienced and highly skilled.

As can be seen in Figure 6, the plotted points tend to be located along a positive linear curve, and the point indicated at the upper right corresponds to the NFG (area N in the figure) whereas the points in the lower left correspond to the SFG (area S). Moreover, there is a point $\mathrm{A}$ in the upper left part of the figure and there are points $\mathrm{B}$ in the lower right part. The factories that fell into the A and B groups are thought to have been assigned services that differ greatly from those assigned to the others.

According to the hypothesis, two factories that have the same average value of Total Test Time are easily identifiable from the others. Factory A and Factory C in Figure 6 are such factories. Factory $A$ is thought to have many test cases of difficult services, and Factory $\mathrm{C}$ is thought to have many unskilled engineers.

Each of the factories is expected to evolve into an advanced one through experience. The evolution stage of each of the factories can be visually assessed by using RPE vs. Total Test Time value and plotting them as shown in Figure 6. Efficient development plans which will increase the efficiency of the test phase in each of the factories can then be worked out.

\section{Conclusion}

With a view to measuring the MM of test cases of new services in the test phase, four factors that characterize the services are identified and used in measuring many actual test cases. By analyzing the information thus obtained, we developed a formula for predicting the MM of test cases, effective curricula in the test phase, and a model for the efficient evolution of each of the factories in the test phase. 
The following three areas need to be further improved:

(a) measurement in the design phase,

(b) successive measurement in the test phase, and

(c) detailed analysis of the measurement results.

Because only a part of the entire development process is measured, serious problems may be transferred to other phases without being recognized. Therefore, all the phases should be targeted for measurement. In that sense, this study should be furthered to find satisfactory solutions for enhancing the productivity of the test phase of large scale software.

\section{Acknowledgments}

Throughout the entire period of this study, we have greatly benefitted from the advice and suggestions of Mr. Ishii, Executive Vice President and Director of the NTT Telecommunications Software Headquarters, and of Mr. Yamaguchi, Executive Manager. Mr. Shidara, Project Manager, also was instrumental in conducting the study by providing us with very helpful advice and direction. Finally, without the support and cooperation of all the testing engineers in all the factories, this study could not have been accomplished, therefore, we would like to express our sincere appreciation to them.

\section{References}

Ishii, K. and Goto, T. (1991) Development Telecommunication Software. NTT R\&D, Vol. 40, No. 11, (In Japanese).

Walston, C. E. and Felix, C. P. (1977) A method of programming measurement and estimation. IBM Systems Journal, Vol. 16, No. 1.

DeMarco, T. (1982) Controlling Software Projects. Youhan Inc., New York.

Beizer, B. (1990) Software Testing Techniques. Van Nostrand Reinhold.

McCabe, T. A. (1976) A Complexity measure. IEEE Transactions on Software Engineering, Vol. 2.

Jones, C. (1991) Applied Software Measurement. McGraw-Hill Professional Publishing Group.

Boehm, B. W. (1981) Software Engineering Economics. Englewood Cliffs, N. J., Prentice-Hall.

Honda, M. (1993) Actual Multivariable Analysis for Information Analysts. Sannou Univ. Print, (In Japanese).

Bergman, M. (1991) The Evolution of Software Testing Automation. Testing Computer Software Proceedings, June 17-20.

Tomono, K., Takeuchi, E., and Tokoyoda, T. (1991) Functioned Technology for Development of Telecommunications. NTT $R \& D$, Vol. 40, No. 11, (In Japanese).

\section{Biography}

Eiji Obara (head author): Bachelor of Science, University of Tokyo, 1988. Assistant Manager, Telecommunications Software Headquarters, NTT. As a team leader, the author has been working on software engineering in the test phase of D70, one of the major switching machines of the NTT network, for six years. He is a member of the Institute of Electronics, Information, and Communication Engineers. 